\title{
Avaliação de Aprendizagem de Machine Learning na Educação Básica: Um Mapeamento da Literatura
}

\author{
Gustavo de Castro Salvador ${ }^{1}$, Christiane Gresse von Wangenheim ${ }^{1}$, Marcelo \\ Fernando Rauber ${ }^{1,2}$, Abisague Belem Garcia ${ }^{1}$, Adriano F. Borgatto ${ }^{1}$ \\ ${ }^{1}$ Departamento de Informática e Estatística \\ Universidade Federal de Santa Catarina (UFSC) - Florianópolis - SC - Brasil \\ ${ }^{2}$ Instituto Federal Catarinense (IFC) - campus Camboriú - SC - Brasil \\ gustavo.castro.salvador@grad.ufsc.br, c.wangenheim@ufsc.br, \\ marcelo.rauber@ifc.edu.br, abisague.garcia@posgrad.ufsc.br, \\ adriano.borgatto@ufsc.br
}

\begin{abstract}
Machine learning (ML) is being increasingly integrated into our life. Thus, to demystify ML several instructional units are emerging teaching $M L$ already in K-12. Yet, especially the assessment of the learning of ML concepts remains an open issue. Thus, in order to provide an overview on the current state of the art regarding the assessment of the learning of $M L$ in $K-12$, we performed a systematic mapping. We identified 15 instructional units on $M L$, which also present the assessment of the students' learning, mostly in a simple manner by few test questions/quizzes with few proposing performance-based assessments. However, an evident lack of systematic definition and validation of these assessments indicates the need for further research.
\end{abstract}

Resumo. Machine Learning (ML) está cada vez mais integrada em nossa vida. Assim, para desmistificar ML, várias unidades instrucionais estão emergindo ensinando ML já na Educação Básica. No entanto, especialmente a avaliação da aprendizagem dos conceitos de ML continua sendo uma questão aberta. Assim, a fim de fornecer uma visão geral sobre o atual estado da arte em relação à avaliação da aprendizagem de ML na Educação Básica, realizamos um mapeamento sistemático. Identificamos 15 unidades instrucionais abordando $M L$, que também apresentam a avaliação da aprendizagem dos estudantes, principalmente de uma maneira simples como testes/questionários com poucos propondo uma avaliação baseada em desempenho. No entanto, há uma evidente falta de definição sistemática e validação dessas avaliações o que indica a necessidade de pesquisas futuras.

\section{Introdução}

Machine Learning (ML) ou Aprendizado de Máquina tornou-se parte de nossa vida cotidiana, impactando profundamente nossa sociedade ao servir como motor de inovação para uma ampla gama de aplicativos, como sistemas de reconhecimento de voz, assistentes inteligentes, carros autônomos, etc. No entanto, a maioria das pessoas não entende a tecnologia por trás disso, o que pode tornar o ML um assunto misterioso ou até assustador, ofuscando seu potencial de impactar positivamente a sociedade [Evangelista et al., 2018][Ho e Scadding, 2019]. 
Assim, para desmistificar o Machine Learning é importante introduzir conceitos e práticas básicas já na escola, permitindo que os estudantes se tornem não apenas consumidores, mas também criadores de soluções inteligentes [Touretzky et al., 2019][Kandlhofer et al., 2016]. A exposição a este conteúdo complexo pode melhorar as habilidades do dia a dia dos estudantes, equipando-os com conhecimento para lidar com as questões, sociais, econômicas e éticas criadas pelo uso de ML nas diversas esferas da sociedade [Kahn et al., 2020]. Também pode encorajar estudantes a considerarem carreiras em Computação e fornecer uma preparação sólida para o ensino superior [CSTA, 2011]. O Machine Learning, como um subcampo da Computação, tem sido tradicionalmente ensinado apenas no ensino superior [Torrey, 2012][McGovern et al., 2011]. E, embora hoje existam muitas iniciativas que têm como foco o ensino de programação e robótica, o ensino de Inteligência Artificial e ML para estudantes da Educação Básica está muito ausente [Hubwieser et al., 2015].

No entanto, recentemente, algumas iniciativas e projetos que buscam ensinar ML na Educação Básica surgiram. Isto inclui diretrizes curriculares, p.ex., pela AI4K12 [Touretzky et al., 2019] e unidades instrucionais, principalmente extracurriculares [Marques et al., 2020]. Essas unidades instrucionais ensinam competências que vão de apresentações sobre o que é ML a técnicas específicas da área, com ênfase em redes neurais artificiais e impactos do ML até a aplicação e criação de modelos de Deep Learning. Poucas unidades instrucionais abordam o processo de ML de forma mais completa, com a maioria abordando somente uma parte, como preparação de dados, ou apresentação de alguns processos apenas de forma abstrata, por exemplo, o treinamento de redes neurais [Marques et al., 2020]. Tipicamente são adotadas ferramentas visuais e/ou ambientes textuais como Jupyter Notebooks, às vezes de forma integrada com ambientes de programação baseado em blocos, como Scratch, SNAP! ou App Inventor [Gresse von Wangenheim et al., 2020]. As unidades instrucionais variam também de unidades escolares e cursos no modo presencial até cursos e tutoriais on-line.

Como parte do processo de ensino, é importante avaliar a aprendizagem dos estudantes fornecendo feedback tanto para o aluno quanto para o professor [Hattie e Timperley, 2007]. No entanto, apesar de pesquisas existentes para avaliação do ensino de pensamento computacional e programação na Educação Básica [Tang et al., 2019][Lye e Koh, 2014][Oliveira e Oliveira, 2014] observa-se a falta de propostas de avaliações da aprendizagem de ML do aluno [Marques et al., 2020] como revisões sistemáticas, como p.ex. Camada e Durães (2020), Sanusi e Oyelere (2020), Zhou et al. (2020) não analisam de forma explícita esta questão. Portanto, o presente artigo visa levantar o estado da arte referente a avaliações da aprendizagem de ML de estudantes da Educação Básica por meio de um mapeamento sistemático da literatura.

\section{Definição e execução do estudo de mapeamento sistemático}

A fim de levantar o estado da arte é realizado um mapeamento sistemático da literatura seguindo o procedimento definido por Petersen et al. (2015).

\subsection{Definição do Protocolo de Revisão}

Pergunta de pesquisa. Quais modelos existem para avaliação de aprendizagem de Machine Learning do aluno na Educação Básica? Esta questão é refinada nas seguintes perguntas de análise: 
AQ1. Quais unidades instrucionais voltadas ao ensino de ML na Educação Básica apresentam avaliações de aprendizagem?

AQ2. Quais são as características destas avaliações em termos de nível de aprendizagem, conteúdo e tipo?

AQ3. Que feedback instrucional é apresentado?

Critérios de seleção: Conforme o foco da pesquisa, são selecionados artigos relevantes seguindo os critérios de inclusão e exclusão apresentados no Quadro 1.

Quadro 1. Critérios de inclusão e exclusão utilizados nesta pesquisa.

\begin{tabular}{|l|l|}
\hline Critérios de inclusão & Critérios de exclusão \\
\hline - Artigos escritos na língua inglesa. & - Artigos que enfocam o ensino de $M L$ no Ensino Infantil \\
- Artigos que apresentem avaliações de aprendizagem de & e/ou Ensino Superior e/ou abordam Inteligência Artificial \\
Machine Learning no contexto da Educação Básica. & sem abordar conceitos de ML. \\
- Artigos publicados nos últimos dez anos de 2011 a 2021, & - Artigos que somente apresentam uma avaliação do \\
considerando a tendência recente de ensino de conceitos & curso, mas não apresentam propostas para a avaliação da \\
de ML. & $\begin{array}{l}\text { aprendizagem do aluno. } \\
\text { - Publicações como blogs, vídeos ou outras ferramentas } \\
\text { que não compõem uma unidade instrucional/curso para }\end{array}$ \\
& $\begin{array}{l}\text { estudantes de Ensino Educação Básica. } \\
\text { - Artigos que não apresentam informações substanciais, } \\
\text { como artigos-resumo ou apenas de uma página. }\end{array}$ \\
\hline
\end{tabular}

Fontes. A busca é feita nos principais repositórios digitais no campo da Computação, incluindo Scopus, IEEE Xplore, ACM, SpringerLink, ScienceDirect, arXiv, SocArXiv, Google Scholar e MIT Media Lab. Além disso, foram realizadas buscas no Google Scholar e Google para complementar a pesquisa, minimizando o risco da omissão [Piasecki et al., 2018]. Buscou-se também publicações no repositório do MIT Media Lab pela atuação nesta área.

String de busca. Com base na pergunta de pesquisa, foram realizadas pesquisas informais para calibrar a string de busca, identificando termos de pesquisa relevantes e sinônimos. Após obter poucos resultados relevantes com uma busca muito específica sobre avaliação em ML na Educação Básica, a estratégia de busca foi alterada visando em primeiro momento a busca por artigos que apresentassem unidades instrucionais voltadas ao ensino de ML, e em seguida considerados relevantes durante o processo de seleção somente artigos que incluíssem uma apresentação de avaliações. Para a busca de unidades instrucionais de ensino de ML foi definido a string seguindo Marques et al. (2020):

(teach* OR education OR course OR MOOC OR learn*) AND ("machine learning" OR "data science” OR "artificial intelligence” OR “deep learning”) AND (“k-12” OR school* OR kids OR children OR teen*)

Observando também basicamente a inexistência de pesquisa voltada ao ensino de ML na Educação Básica em português, foi adotado somente um string de busca em inglês.

\subsection{Execução da busca}

A busca adaptada da search string a cada repositório foi realizada nos títulos e resumos limitada às áreas de Computação e Educação pelos autores em conjunto em março de 2021, conforme apresentado no Quadro 2. 
Quadro 2. Número de artigos identificados por repositório e por fase de seleção

\begin{tabular}{|l|r|l|l|l|}
\hline Fonte & $\begin{array}{l}\text { No. de resultados } \\
\text { da busca }\end{array}$ & $\begin{array}{l}\text { No. de resultados } \\
\text { analisados }\end{array}$ & $\begin{array}{l}\text { No. de documentos } \\
\text { potencialmente } \\
\text { relevantes }\end{array}$ & $\begin{array}{l}\text { No. de documentos } \\
\text { relevantes }\end{array}$ \\
\hline Scopus & 3.760 & 500 & 37 & 0 \\
\hline IEEE Explore & 1.113 & 500 & 11 & 4 \\
\hline ACM & 4.151 & 500 & 25 & 0 \\
\hline SpringerLink & 47.718 & 500 & 4 & 0 \\
\hline ScienceDirect & 57 & 57 & 1 & 3 \\
\hline arXiv & 140 & 140 & 4 & 1 \\
\hline SocArXiv & 7.404 & 500 & 4 & 3 \\
\hline Google Scholar & 17.000 & 500 & 11 & 12 \\
\hline Google & 633.000 & 500 & 45 & 10 \\
\hline MIT Media Lab ${ }^{1}$ & 81 & 81 & Total & $\mathbf{1 5}$ (sem duplicatas) \\
\hline
\end{tabular}

A partir do resultado das buscas, foram selecionados artigos potencialmente relevantes de acordo com os critérios de inclusão e exclusão por meio de uma análise do título, resumo e palavra-chave de cada artigo. Foram analisados os primeiros 500 artigos mais relevantes encontrados em cada busca. Em seguida, foram analisados os artigos potencialmente relevantes pela sua leitura na íntegra. Muitos artigos se referem ao uso de técnicas de IA/ML na educação ao invés de ensinar ML. Estes foram excluídos, assim como artigos voltados a outros estágios educacionais e/ou artigos que apresentavam unidades instrucionais para ensinar ML, porém sem demonstrar algum tipo de avaliação da aprendizagem do aluno. Eliminando as duplicatas foram identificadas 15 unidades instrucionais relevantes como resultado final, conforme apresentado no Quadro 3. Como nem todas as unidades instrucionais disponibilizam explicitamente as informações desejadas, certas características foram inferidas pelos pesquisadores de acordo com a informação disponível nos próprios artigos e/ou cursos/plataformas educativas.

\section{Resultados}

\subsection{Quais avaliações de aprendizagem de ML na Educação Básica existem?}

\section{Quadro 3. Artigos relevantes}

\begin{tabular}{|l|l|l|l|}
\hline \multicolumn{1}{|c|}{ ID } & \multicolumn{1}{|c|}{ Nome } & \multicolumn{1}{|c|}{ Descrição } & \multicolumn{1}{|c|}{ Referência } \\
\hline $\begin{array}{l}\text { [Al Family } \\
\text { Challenge, 2019] }\end{array}$ & $\begin{array}{l}\text { Al Family } \\
\text { Challenge }\end{array}$ & $\begin{array}{l}\text { Desafio para famílias aprenderem sobre IA e ML } \\
\text { e resolver um problema em suas comunidades } \\
\text { usando ferramentas de IA. }\end{array}$ & $\begin{array}{l}\text { https://www.curiositymachine } \\
\text { org/about/ }\end{array}$ \\
\hline $\begin{array}{l}\text { [Apps For Good, } \\
2019]\end{array}$ & $\begin{array}{l}\text { Apps for Good: ML } \\
\text { course }\end{array}$ & $\begin{array}{l}\text { Curso que fornece uma visão geral de tópicos de } \\
\text { ML e permite às equipes de estudantes projetar } \\
\text { e construir um protótipo que resolva um } \\
\text { problema real usando algoritmos de ML. }\end{array}$ & $\begin{array}{l}\text { https://www.appsforgood.org } \\
\text { /courses/machine-learning }\end{array}$ \\
\hline $\begin{array}{l}\text { [Brummelen et al., } \\
\text { 2020] }\end{array}$ & $\begin{array}{l}\text { Al Literacy } \\
\text { Workshop } \\
\text { desenvolvem agentes de conversação usando } \\
\text { uma interface no MIT App Inventor. }\end{array}$ & $\begin{array}{l}\text { J. V. Brummelen et al. } \\
\text { Teaching Tech to Talk: K-12 } \\
\text { Conversational Artificial } \\
\text { Intelligence Literacy } \\
\text { Curriculum and } \\
\text { Development Tools. arXiv, } \\
\text { 2020. }\end{array}$ \\
\hline [Code.org, 2019] & Al for Oceans & $\begin{array}{l}\text { Curso on-line de introdução a ML envolvendo } \\
\text { classificação de peixes do oceano como parte }\end{array}$ & $\begin{array}{l}\text { https://curriculum.code.org/h } \\
\text { oc/plugged/9/ }\end{array}$ \\
\hline
\end{tabular}

1https://appinventor.mit.edu/explore/research 


\begin{tabular}{|c|c|c|c|}
\hline & & dos cursos da code.org. & \\
\hline [CSER, 2020] & $\begin{array}{l}\text { Teaching Artificial } \\
\text { Intelligence in the } \\
\text { Secondary } \\
\text { Classroom }\end{array}$ & $\begin{array}{l}\text { Curso gratuito de IA voltado para instrutores de } \\
\text { IA usando ML em reconhecimento de imagens e } \\
\text { visão computacional. }\end{array}$ & $\begin{array}{l}\text { https://csermoocs.appspot.c } \\
\text { om/ai_secondary/ }\end{array}$ \\
\hline $\begin{array}{l}\text { [Curiosity } \\
\text { Machine, 2019] }\end{array}$ & $\begin{array}{l}\text { Curiosity Machine } \\
\text { - build a } \\
\text { neural network }\end{array}$ & $\begin{array}{l}\text { Desafio para construir um protótipo de uma rede } \\
\text { neural artificial desconectada para classificação } \\
\text { de diferentes objetos. }\end{array}$ & $\begin{array}{l}\text { https://www.curiositymachine } \\
\text {.org/challenges/126/ }\end{array}$ \\
\hline $\begin{array}{l}\text { [Elements of Al, } \\
\text { 2019] }\end{array}$ & Elements of $\mathrm{Al}$ & $\begin{array}{l}\text { Curso on-line voltado ao ensino de ML para } \\
\text { aprender o que é Inteligência Artificial, o que } \\
\text { pode (e não pode) ser feito com IA e como } \\
\text { começar a criar métodos de IA, inclusive ML. }\end{array}$ & $\begin{array}{l}\text { https://course.elementsofai.c } \\
\text { om/4 }\end{array}$ \\
\hline $\begin{array}{l}\text { [Exploring } \\
\text { Computer } \\
\text { Science, 2020] }\end{array}$ & $\begin{array}{l}\text { Alternate } \\
\text { Curriculum Unit: Al }\end{array}$ & $\begin{array}{l}\text { Curso que leva os estudantes a explorarem } \\
\text { aplicações práticas no dia a dia da IA e entender } \\
\text { o impacto da IA em suas vidas por meio de } \\
\text { diversas atividades. }\end{array}$ & $\begin{array}{l}\text { http://www.exploringcs.org/w } \\
\text { p- } \\
\text { content/uploads/2019/09/AI- } \\
\text { Unit-9-16-19.pdf }\end{array}$ \\
\hline $\begin{array}{l}{[\text { Gresse von }} \\
\text { Wangenheim et } \\
\text { al., 2020] }\end{array}$ & \begin{tabular}{|l} 
Machine Learning \\
para Todos!
\end{tabular} & $\begin{array}{l}\text { Curso que introduz conceitos básicos de ML, } \\
\text { levando o aluno a criar um modelo de } \\
\text { reconhecimento de imagens para separação de } \\
\text { lixo reciclável usando o Google Teachable } \\
\text { Machine. }\end{array}$ & $\begin{array}{l}\text { C. Gresse von Wangenheim, } \\
\text { et al. Machine Learning for } \\
\text { All - Introducing Machine } \\
\text { Learning in K-12. SocArXiv, } \\
2020 .\end{array}$ \\
\hline [Lee et al., 2021] & \begin{tabular}{|l} 
Developing Middle \\
School Students' \\
Al Literacy
\end{tabular} & $\begin{array}{l}\text { Oficina de verão para preparar estudantes do } \\
\text { Ensino Médio a desenvolver conhecimentos e } \\
\text { habilidades fundamentais sobre IA e tornarem } \\
\text { cidadãos informados e consumidores críticos da } \\
\text { tecnologia. }\end{array}$ & $\begin{array}{l}\text { Lee, I. et al. Developing } \\
\text { Middle School Students' AI } \\
\text { Literacy. ACM Special } \\
\text { Interest Group on Computer } \\
\text { Science Education, 2021. } \\
\end{array}$ \\
\hline $\begin{array}{l}\text { [MIT App Inventor, } \\
\text { 2019] }\end{array}$ & $\begin{array}{l}\text { Introduction to } \\
\text { Machine Learning: } \\
\text { Image } \\
\text { Classification }\end{array}$ & $\begin{array}{l}\text { Curso que ensina noções básicas de ML e a } \\
\text { criação de aplicativos que implementam esses } \\
\text { conceitos por meio de classificação de imagens } \\
\text { pelos estudantes com deployment via apps com } \\
\text { App Inventor. }\end{array}$ & $\begin{array}{l}\text { https://appinventor.mit.edu/e } \\
\text { xplore/resources/ai/image- } \\
\text { classification-look-extension }\end{array}$ \\
\hline [ReadyAl, 2019] & ReadyAl Al + Me & $\begin{array}{l}\text { Curso on-line destinado a fornecer aos } \\
\text { estudantes os conceitos básicos de IA e de ML. }\end{array}$ & $\begin{array}{l}\text { https://edu.readyai.org/cours } \\
\text { es/aime/ }\end{array}$ \\
\hline $\begin{array}{l}\text { [Rodríguez-García } \\
\text { et al., 2021] }\end{array}$ & LearningML & $\begin{array}{l}\text { Oficina que ensina Machine Learning no } \\
\text { contexto de desenvolvimento de projetos Scratch } \\
\text { fornecendo uma plataforma web para o ensino } \\
\text { de fundamentos de ML, permitindo treinar e } \\
\text { testar modelos on-line e fazer o deployment via } \\
\text { Scratch. }\end{array}$ & $\begin{array}{l}\text { J. D. Rodríguez García et al. } \\
\text { Evaluation of an Online } \\
\text { Intervention to Teach } \\
\text { Artificial Intelligence With } \\
\text { LearningML to 10-16-Year- } \\
\text { Old Students. Technical } \\
\text { Symposium of the ACM } \\
\text { Special Interest Group on } \\
\text { Computer Science } \\
\text { Education, 2021. }\end{array}$ \\
\hline $\begin{array}{l}\text { [Sakulkueakulsuk } \\
\text { et al., 2018] }\end{array}$ & Kids making Al & $\begin{array}{l}\text { Desafio de IA baseado em agricultura que visa } \\
\text { ensinar o processo de criação de modelos de } \mathrm{ML} \\
\text { na forma de um jogo. }\end{array}$ & $\begin{array}{l}\text { B. Sakulkueakulsuk et al. } \\
\text { Kids making Al: Integrating } \\
\text { Machine Learning, } \\
\text { Gamification, and Social } \\
\text { Context in STEM Education. } \\
\text { Proc. of IEEE Int. Conf. on } \\
\text { Teaching, Assessment, and } \\
\text { Learning for Engineering, } \\
\text { Wollongong, Australia, 2018. }\end{array}$ \\
\hline [Tang, 2019] & $\begin{array}{l}\text { Personal Image } \\
\text { Classifier }\end{array}$ & $\begin{array}{l}\text { Curso que ensina noções básicas de ML e faz } \\
\text { com que os estudantes criem seus próprios } \\
\text { aplicativos que implementam esses conceitos } \\
\text { por meio de classificação de imagens com } \\
\text { deployment via apps com App Inventor. }\end{array}$ & $\begin{array}{l}\text { D. Tang. Empowering } \\
\text { Novices to Understand and } \\
\text { Use Machine Learning With } \\
\text { Personalized Image } \\
\text { Classification Models, } \\
\text { Intuitive Analysis Tools, and } \\
\text { MIT App Inventor. Master } \\
\text { thesis, MIT, USA, 2019. }\end{array}$ \\
\hline
\end{tabular}

Foram encontradas 15 unidades instrucionais relevantes (Quadro 4) que apresentam algum tipo de avaliação de aprendizagem de ML voltado a estudantes da Educação Básica. Observa-se que mesmo tendo uma quantidade considerável de cursos 
de $M L$ para o Educação Básica (Marques et al., 2020)(Camada e Durães, 2020), poucos abordam de forma explícita a avaliação da aprendizagem do aluno. A maioria visa o ensino de ML especificamente ou como parte da Inteligência Artificial de forma geral ao nível da Educação Básica. Poucas são especificamente voltadas ao Ensino Fundamental ou Ensino Médio.

\subsection{Quais são as características destas avaliações?}

Quadro 4. Características das avaliações

\begin{tabular}{|c|c|c|c|c|}
\hline ID & Método de avaliação & $\begin{array}{l}\text { Níveis de aprendizagem } \\
\text { do Bloom }\end{array}$ & $\begin{array}{l}\text { Fases do } \\
\text { "Use- } \\
\text { Modify- } \\
\text { Create" }\end{array}$ & $\begin{array}{l}\text { Conceitos de ML } \\
\text { avaliados }\end{array}$ \\
\hline $\begin{array}{l}\text { [Al Family } \\
\text { Challenge, 2019] }\end{array}$ & $\begin{array}{l}\text { Quiz com uma questão de } \\
\text { múltipla escolha }\end{array}$ & $\begin{array}{l}\text { Conhecimento, } \\
\text { Compreensão, Aplicação }\end{array}$ & Use & $\begin{array}{l}\text { Conceitos básicos de } \\
\text { ML }\end{array}$ \\
\hline $\begin{array}{l}\text { [Apps For Good, } \\
\text { 2019] }\end{array}$ & Questões de avaliação & $\begin{array}{l}\text { Conhecimento, } \\
\text { Compreensão, Aplicação }\end{array}$ & Use, Create & $\begin{array}{l}\text { Conceitos básicos de } \\
\text { ML, impacto de ML }\end{array}$ \\
\hline \begin{tabular}{|l} 
[Brummelen et al., \\
2020]
\end{tabular} & $\begin{array}{l}\text { Questões de avaliação, } \\
\text { avaliação baseado em } \\
\text { desempenho de ideas }\end{array}$ & $\begin{array}{l}\text { Conhecimento, } \\
\text { Compreensão e Aplicação }\end{array}$ & \begin{tabular}{|l|} 
Use, Modify, \\
Create
\end{tabular} & $\begin{array}{l}\text { Conceitos básicos de } \\
\text { ML, impacto de ML }\end{array}$ \\
\hline [Code.org, 2019] & Conclusão da tarefa & $\begin{array}{l}\text { Conhecimento, } \\
\text { Compreensão }\end{array}$ & ---- & $\begin{array}{l}\text { Conceitos básicos de } \\
\text { ML }\end{array}$ \\
\hline [CSER, 2020] & Questões abertas/de redação & $\begin{array}{l}\text { Conhecimento, } \\
\text { Compreensão }\end{array}$ & Use & $\begin{array}{l}\text { Conceitos básicos de } \\
\mathrm{ML}\end{array}$ \\
\hline $\begin{array}{l}\text { [Curiosity } \\
\text { Machine, 2019] }\end{array}$ & $\begin{array}{l}\text { Quiz com } 3 \text { questões de múltipla } \\
\text { escolha }\end{array}$ & $\begin{array}{l}\text { Conhecimento, } \\
\text { Compreensão }\end{array}$ & --- & Redes neurais \\
\hline $\begin{array}{l}\text { [Elements of Al, } \\
\text { 2019] }\end{array}$ & $\begin{array}{l}\text { Exercícios com 1-3 questões } \\
\text { abertas/de redação ou de } \\
\text { múltipla escolha }\end{array}$ & $\begin{array}{l}\text { Conhecimento, } \\
\text { Compreensão }\end{array}$ & ---- & $\begin{array}{l}\text { Conceitos básicos de } \\
\text { ML, impacto de ML, } \\
\text { redes neurais }\end{array}$ \\
\hline $\mid \begin{array}{l}\text { [Exploring } \\
\text { Computer } \\
\text { Science, 2020] }\end{array}$ & $\begin{array}{l}\text { Rubricas com critérios com } 8 \text { - } \\
14 \text { para a avaliação das } \\
\text { apresentações dos estudantes, } \\
\text { questões de redação }\end{array}$ & $\begin{array}{l}\text { Conhecimento, } \\
\text { Compreensão }\end{array}$ & ---- & $\begin{array}{l}\text { Conceitos básicos de } \\
\text { ML, impacto de } M L\end{array}$ \\
\hline $\begin{array}{l}\text { [Gresse von } \\
\text { Wangenheim et } \\
\text { al., 2020] }\end{array}$ & $\begin{array}{l}\text { Quiz, questões verdadeiro/falso, } \\
\text { arrastar e soltar, rubrica de } 11 \\
\text { critérios para a avaliação dos } \\
\text { artefatos do desenvolvimento de } \\
\text { um modelo de ML }\end{array}$ & $\begin{array}{l}\text { Conhecimento, } \\
\text { Compreensão, Aplicação }\end{array}$ & Use & $\begin{array}{l}\text { Conceitos básicos de } \\
\text { ML, redes neurais, } \\
\text { processo de } M L \\
\text { impacto de } M L\end{array}$ \\
\hline [Lee et al., 2021] & $\begin{array}{l}\text { Quiz, questões verdadeiro/falso, } \\
\text { questões abertas/de redação }\end{array}$ & $\begin{array}{l}\text { Conhecimento, } \\
\text { Compreensão } \\
\end{array}$ & Use, Modify & $\begin{array}{l}\text { Conceitos básicos de } \\
\text { ML, redes neurais }\end{array}$ \\
\hline $\begin{array}{l}\text { [MIT App Inventor, } \\
\text { 2019] }\end{array}$ & $\begin{array}{l}\text { Teste com } 3 \text { questões de } \\
\text { múltipla escolha }\end{array}$ & $\begin{array}{l}\text { Conhecimento, } \\
\text { Compreensão, Aplicação }\end{array}$ & Use & Redes neurais \\
\hline [ReadyAl, 2019] & $\begin{array}{l}\text { Quiz de pergunta única (múltipla } \\
\text { escolha, arrastar e soltar, etc.) }\end{array}$ & $\begin{array}{l}\text { Conhecimento, } \\
\text { Compreensão }\end{array}$ & --- & $\begin{array}{l}\text { Conceitos básicos de } \\
\text { ML, impacto de ML }\end{array}$ \\
\hline $\begin{array}{l}\text { [Rodríguez-García } \\
\text { et al., 2021] }\end{array}$ & $\begin{array}{l}\text { Quiz com } 14 \text { questões, } \\
\text { exercícios, questão aberta }\end{array}$ & $\begin{array}{l}\text { Conhecimento, } \\
\text { Compreensão, Aplicação }\end{array}$ & \begin{tabular}{|l|} 
Use, Modify, \\
Create
\end{tabular} & $\begin{array}{l}\text { Conceitos básicos de } \\
\text { ML, redes neurais }\end{array}$ \\
\hline $\begin{array}{l}\text { [Sakulkueakulsuk } \\
\text { et al., 2018] }\end{array}$ & $\begin{array}{l}\text { Gamificação de pontos de } \\
\text { acordo com a performance dos } \\
\text { modelos de ML }\end{array}$ & $\begin{array}{l}\text { Conhecimento, } \\
\text { Compreensão }\end{array}$ & Use & $\begin{array}{l}\text { Conceitos básicos de } \\
\mathrm{ML}\end{array}$ \\
\hline [Tang, 2019] & $\begin{array}{l}\text { Teste com } 3 \text { questões de } \\
\text { múltipla escolha }\end{array}$ & $\begin{array}{l}\text { Conhecimento, } \\
\text { Compreensão, Aplicação }\end{array}$ & Use & Rede \\
\hline
\end{tabular}

A maioria das avaliações são relativamente simples, referindo-se somente à aprendizagem nos níveis de conhecimento e compreensão, seguindo a Taxonomia de Bloom et al. (1965), com poucas que abordam também o nível de aplicação. Como o foco da maioria dos cursos no nível introdutório, a aprendizagem de conceitos básicos de ML é a mais avaliada, menos frequentemente avalia-se a aprendizagem de conceitos de redes neurais. Várias avaliações abordam questões éticas e o impacto de ML na sociedade. 
Em alguns casos, as avaliações consistem somente em questões de resposta única no final das unidades instrucionais ou apenas monitoram a conclusão da tarefa [Code.org, 2019]. Algumas unidades avaliam as respostas dos estudantes a exercícios [Elements of AI, 2019]. Várias unidades instrucionais usam quizzes com diferentes tipos de questões (verdadeiro/falso, múltipla escolha, arrastar e soltar, etc.), incluindo por exemplo, [Gresse von Wangenheim et al., 2020][ReadyAI, 2019][Rodríguez-García et al., 2021], enquanto MIT App Inventor (2019) e Tang (2019) utilizam testes com 3 questões de múltipla escolha. Ao levar em consideração que a maioria das unidades instrucionais são usualmente oferecidas como atividades extracurriculares, estes tipos de avaliações simples são adequados para prevenir a desmotivação dos estudantes. Entretanto, a falta de avaliações mais rigorosas pode impedir um melhor suporte para a aprendizagem dos estudantes e o aprimoramento das unidades instrucionais.

Poucas unidades adotam uma avaliação baseada em desempenho definindo rubricas para a avaliação de apresentações dos projetos [Exploring Computer Science, 2020] ou rubricas para avaliar artefatos de modelos de ML criados pelos estudantes [Gresse von Wangenheim et al., 2020]. Dado a natureza recente das unidades instrucionais de ML para a Educação Básica, a maioria foca na avaliação de resultados na fase de Use do ciclo "Use-Modify-Create". Há exceções, como as unidades propostas por Rodríguez-García et al. (2021) e Apps for Good (2019), levando os estudantes a criarem modelos personalizados de ML. Essa criação é feita adotando a estratégia de "ação computacional" [Tissenbaum et al., 2019], que permite que os estudantes aprendam enquanto criam artefatos significativos que têm um impacto em suas vidas e comunidades que estejam inseridos.

\subsection{Que feedback instrucional é apresentado?}

Quadro 6. Tipos de feedback apresentados

\begin{tabular}{|l|l|l|}
\hline \multicolumn{1}{|c|}{ ID } & \multicolumn{1}{|c|}{ Tipo de feedback } & \multicolumn{1}{c|}{ Tipo de automação } \\
\hline [Al Family Challenge, 2019] & Correção de respostas & Correção automatizada de respostas \\
\hline [Apps For Good, 2019] & Avaliação manual pelo instrutor & Não possui \\
\hline [Brummelen et al., 2020] & Avaliação manual pelo instrutor & Não possui \\
\hline [Code.org, 2019] & $\begin{array}{l}\text { Indicação de conclusão da tarefa sem análise } \\
\text { de correção. Feedback para instrutores para } \\
\text { monitoramento de uma turma }\end{array}$ & Grau de conclusão da tarefa \\
\hline [CSER, 2020] & $\begin{array}{l}\text { Comparação manual por parte do aluno de um } \\
\text { modelo ideal fornecido pelo instrutor }\end{array}$ & Não possui \\
\hline [Curiosity Machine, 2019] & Apresentação das respostas fornecidas & Não possui \\
\hline [Elements of Al, 2019] & $\begin{array}{l}\text { Correção de respostas de múltipla escolha, } \\
\text { apresenta respostas de exemplo e revisão por } \\
\text { pares para respostas de texto. Há certificado, e } \\
\text { a quantidade de exercícios realizados é } \\
\text { monitorada }\end{array}$ & $\begin{array}{l}\text { Correção automatizada de respostas de } \\
\text { múlipla escolha }\end{array}$ \\
\hline [Exploring Computer & $\begin{array}{l}\text { Avaliação manual pelo instrutor indicando o } \\
\text { total de pontos }\end{array}$ & Não possui \\
\hline Science, 2020] & $\begin{array}{l}\text { Correção de respostas, acompanhamento de } \\
\text { conclusão de tarefas } \\
\text { et al., 2020] }\end{array}$ & $\begin{array}{l}\text { Correção automatizada de respostas dos } \\
\text { quizzes, não possui automação para } \\
\text { avaliação da rubrica }\end{array}$ \\
\hline [Lee et al., 2021] & Avaliação manual pelo instrutor & Não possui \\
\hline [MIT App Inventor, 2019] & Avaliação manual pelo instrutor & Não possui \\
\hline [ReadyAl, 2019] & $\begin{array}{l}\text { Correção de respostas, acompanhamento de } \\
\text { conclusão de tarefas }\end{array}$ & Correção automatizada de respostas \\
\hline
\end{tabular}




\begin{tabular}{|c|c|c|}
\hline $\begin{array}{l}\text { [Rodríguez-García et al., } \\
\text { 2021] }\end{array}$ & Autocorreção testando o modelo & $\begin{array}{l}\text { Cálculo de precisão e desempenho do } \\
\text { modelo criado }\end{array}$ \\
\hline $\begin{array}{l}\text { [Sakulkueakulsuk et al., } \\
\text { 2018] }\end{array}$ & Avaliação manual pelo instrutor & Não possui \\
\hline [Tang, 2019] & Avaliação manual pelo instrutor & Não possui \\
\hline
\end{tabular}

O feedback instrucional, tipicamente, é limitado à indicação se as questões foram respondidas corretamente, sem maiores informações. Muitas avaliações não possuem algum tipo de automação para efetuar as avaliações, sendo executadas por instrutores das unidades instrucionais. Basicamente, somente quizzes são automatizados nas unidades on-line. Uma exceção é a avaliação apresentada por Rodríguez-García et al. (2021), que retorna a precisão e desempenho do modelo criado para a avaliação do aluno. A avaliação de Alternate Curriculum Unit: AI [Exploring Computer Science, 2020] utiliza uma escala de pontuação para apresentar o feedback ao estudante com base na soma ponderada dos itens da rubrica, sendo julgado por juízes que representam especialistas do domínio. Ao final, algumas unidades oferecem um certificado para completar o curso e para aumentar o engajamento [Code.org, 2019]. E Sakulkueakulsuk et al. (2018) adotam uma abordagem de gamificação para aumentar a motivação do aluno também no momento da avaliação.

\section{Discussão}

Observa-se como resultado desta revisão que atualmente a maioria das unidades instrucionais de ensino de Machine Learning para estudantes da Educação Básica não apresentam propostas para a avaliação de aprendizagem dos estudantes. Isto pode ser explicado pelo fato que o ensino de ML na Educação Básica ainda está emergente com um aumento de unidades instrucionais principalmente a partir dos últimos dois anos [Marques et al., 2020]. Mesmo vários artigos apresentando avaliações da qualidade de curso por meio de pré/pós-testes, no quesito de avaliação de aprendizagem do aluno usam somente uma autoavaliação não medindo de forma mais confiável a aprendizagem.

O enfoque da maioria dos cursos no nível iniciante explica a ênfase em níveis de aprendizagem mais baixos segundo a Taxonomia de Bloom, com poucos que levam e avaliam a competência ao nível de aplicação de ML. Assim, a maioria das avaliações foca em conceitos básicos a serem avaliados por meio de quizzes e testes. Foram identificados poucos exemplos de rubricas de avaliação de desempenho que analisam artefatos criados pelo aluno ao aplicar conceitos de ML. Outro fator que impede a avaliação de um escopo maior é a típica curta duração destes cursos, muitas vezes de forma extracurricular. O tipo de feedback apresentado para os estudantes costuma ser simples, o qual indica se a tarefa foi concluída ou realizada corretamente. A maioria das avaliações propostas são realizadas manualmente pelos instrutores e/ou juízes. Identificou-se a automação de avaliações somente no caso de cursos on-line referente a quizzes.

Com base nestes resultados é evidente a necessidade de aprimoramento de modelos de avaliação do ensino de ML na Educação Básica, tanto em termos do seu desenvolvimento e validação sistemática quanto uma cobertura maior dos objetivos de aprendizagem e automação para preparar uma futura adoção mais ampla em escolas brasileiras.

Ameaças à validade. Mapeamentos sistemáticos podem sofrer do viés comum de que os resultados positivos têm maior probabilidade de serem publicados do que os negativos. 
No entanto, foi considerado que os resultados, sejam positivos ou negativos, têm apenas uma pequena influência nesse mapeamento, uma vez que foi buscado caracterizar os modelos de avaliação de aprendizagem. A fim de mitigar o risco de omitir algum artigo relevante, a string de busca foi construída para ser o mais abrangente possível, considerando não apenas os principais conceitos, mas também sinônimos e a busca em diversas fontes. Ameaças relacionadas a seleção e extração de dados foram mitigadas por meio da definição detalhada dos critérios de inclusão e exclusão, além da revisão da seleção e extração em conjunto com todos os coautores até que um consenso fosse obtido.

\section{Conclusão}

O presente artigo expõe um mapeamento sistemático sobre abordagens existentes de avaliações de aprendizagem de ensino de Machine Learning no contexto da Educação Básica. Identificamos 15 unidades instrucionais em ML, que também apresentam a avaliação da aprendizagem dos estudantes, a maioria de uma maneira simples, com poucos testes/questionários e poucas propostas de avaliação baseada em desempenho. A maioria das avaliações tem enfoque em níveis introdutórios abrangendo somente conceitos básicos de ML e níveis mais baixos de aprendizagem da Taxonomia de Bloom. O feedback fornecido ao aluno também se restringe à indicação da resposta correta e conclusão da tarefa, sem indicação de informações mais construtivas que guiem o processo de aprendizagem do aluno. A maioria das avaliações também não oferece um suporte automatizado, limita-se basicamente a cursos on-line em relação a correção de respostas de quizzes. Estes resultados demonstram uma falta aparente de avaliações confiáveis e válidas de forma mais completa e abrangente, o que indica a necessidade de pesquisas nesta área para suportar a introdução do ensino de ML de forma mais ampla em escolas brasileiras levando em consideração a democratização desse conhecimento.

\section{Agradecimentos}

O presente trabalho foi realizado com apoio do Conselho Nacional de Desenvolvimento Científico e Tecnológico - Brasil (CNPq).

\section{Referências}

Bloom, B. S.; Engelhart, M. D.; Furst, E. J.; Hill, W. H.; Krathwohl, D. R. Taxonomy of educational objectives. New York: David McKay Company, 1956.

Camada, M. Y.; Durães, G. M. Ensino da Inteligência Artificial na Educação Básica: um novo horizonte para as pesquisas brasileiras. Anais do Simpósio Brasileiro de Informática na Educação, online, 2020.

CSTA. CSTA K-12 Computer Science Standards. ACM, New York, NY, USA, 2011.

Evangelista, I.; Blesio, G.; Benatti, E. Why Are We Not Teaching Machine Learning at High School? A Proposal. World Engineering Education Forum, Albuquerque, NM, USA.,2018.

Galan, D.; Heradio, R.; Vargas, H.; Abad, I.; Cerrada, J. A. Automated Assessment of Computer Programming Practices: The 8-Years UNED Experience. IEEE Access, 7, 2019.

Gresse von Wangenheim, C.; Hauck, J. C. R.; Pacheco, F. S.; Bertonceli Bueno, M. F. Ferramentas Visuais para o Ensino de Machine Learning na Educação Básica. RENOTE, 18(2), 2020. 
Hattie, J; Timperley, H. The power of feedback. Review of Educational Research, 77(1), 2007.

Ho, J. W. K.; Scadding, M. Classroom Activities for Teaching Artificial Intelligence to Primary School Students. Proc. of the Int.Conference on Computational Thinking, Hong Kong, China, 2019.

Hubwieser, P. et al. A Global Snapshot of Computer Science Education in K-12 Schools. Proc. of the ITiCSE on Working Group Reports, Vilnius, Lithuania. 2015.

Kahn, K. M.; Lu, Y., Zhang, J.; Winters, N.; Gao, M. Deep learning programming by all. Proc. of the Conference on Constructionism, Dublin, Ireland. 2020.

Kandlhofer, M.; Steinbauer G.; Hirschmugl-Gaisch S.; Huber, P. Artificial Intelligence and Computer Science in Education. Proc. of the Frontiers in Education Conference, Erie, PA, USA, 2016.

Lye, S. Y.; Koh, J. H. L. Review on teaching and learning of computational thinking through programming: What is next for K-12?. Computers in Human Behavior, 41, 2014.

Marques, L. S.; Gresse von Wangenheim, C.; Hauck, J. C. R. Hauck. Ensino de Machine Learning na Educação Básica: um Mapeamento Sistemático do Estado da Arte. Anais do Simpósio Brasileiro de Informática na Educação, Natal, Brasil, 2020.

McGovern, A.; Tidwell, Z.; Rushing, D. Teaching Introductory Artificial Intelligence through Java-Based Games. Proc. of the 2nd Symposium on Educational Advances in Artificial Intelligence, San Francisco, CA, USA, 2011.

Oliveira, M.; Oliveira, E. Metodologia de Diagnóstico e Regulação de Componentes de Habilidades da Aprendizagem de Programação. Anais do Workshop sobre Educação em Computação, Brasília, Brasil, 2014.

Petersen, K.; Feldt, R.; Mujtaba, S.; Mattsson, M. Systematic Mapping Studies in Software Engineering. Proc. of the 12th Int. Conference on Evaluation and Assessment in Software Engineering, Bari, Italy, 2008.

Piasecki, J.; Waligora, M.; Dranseika, V. Google Search as an Additional Source in Systematic Reviews. Science and Engineering Ethics 24, 2018.

Sanusi, I. T.; Oyelere, S. S. Pedagogies of Machine Learning in K-12 Context. IEEE Frontiers in Education ConferenceUppsala, Sweden, 2020.

Tang, D.; Utsumi, Y.; Lao, N. PIC: A Personal Image Classification Webtool for High School Students. Proc. of the Int. Joint Conferences on Artificial Intelligence, Macao, China, 2019.

Tissenbaum, M.; Sheldon, J.; Abelson, H. From computational thinking to computational action. Communications of the ACM, 62(3), 2019.

Torrey, L. Teaching Problem-Solving in Algorithms and AI. Proc. of the 3rd Symposium on Educational Advances in Artificial Intelligence, Toronto, Ontario, Canada, 2012.

Touretzky, D. S. et al. Envisioning AI for K-12: What Should Every Child Know about AI? Proc. of the 33rd Conf. on Artificial Intelligence, Honolulu, HI, USA, 2019.

Zhou, X.; Brummelen, J. V.; Lin, P. Designing AI Learning Experiences for K-12: Emerging Works, Future Opportunities and a Design Framework, arXiv, 2020. 